\title{
Detection of Treg/Th17 cells and related cytokines in peripheral blood of chronic hepatitis B patients combined with thrombocytopenia and the clinical significance
}

\author{
YINGHUI WANG ${ }^{1,2}$, LIRU WANG $^{2}$, WEI GAO ${ }^{3}, \mathrm{XI} \mathrm{CHEN}^{4}$ and YANHUA SU ${ }^{1}$ \\ ${ }^{1}$ Department of Hematology, The First Affiliated Hospital of Harbin Medical University; Departments of \\ ${ }^{2}$ Hematology and ${ }^{3}$ Infectious Diseases, Heilongjiang Provincial Hospital; ${ }^{4}$ Department of Hematology, \\ The Second Affiliated Hospital of Harbin Medical University, Harbin, Heilongjiang 150036, P.R. China
}

Received August 25, 2017; Accepted May 23, 2018

DOI: $10.3892 / \mathrm{etm} .2018 .6311$

\begin{abstract}
Changes of regulatory $\mathrm{T}$ (Treg) cells and Th17 cells, and related cytokines in peripheral blood of patients with chronic hepatitis B combined with thrombocytopenia were investigated to explore the relationship with treatment outcomes. A total of 45 chronic hepatitis B patients combined with thrombocytopenia were selected in Heilongjiang Provincial Hospital from June 2015 to December 2016. All patients were treated with prednisolone acetate $+\gamma$ globulins for 60 days. Treg cells and Th17 cells in peripheral blood were detected by flow cytometry, and IL-10, TGF- $\beta$, IL-17, IL-21 and IL-22 in peripheral blood were detected by ELISA before and after treatment. No significant differences in the percentages of Treg and Th17, and levels of IL-10, TGF- $\beta$, IL-17, IL-21 and IL-22 were found in nonresponders ( $\mathrm{n}=17$, platelets $\left.<100 \times 10^{9} / \mathrm{l}\right)$ before and after treatment ( $\mathrm{P}>0.05)$. After treatment, percentage of Treg was significantly increased (higher than that of non-responders) and percentage of Th17 was significantly decreased (lower than that of nonresponders) in responders $(\mathrm{P}<0.05)$. In addition, serum levels of IL-10 and TGF- $\beta$ were significantly increased (higher than that of non-responders) and serum levels of IL-17, IL-21 and IL-22 were significantly decreased (lower than that of non-responders) in responders $(\mathrm{P}<0.05)$. The results showed that after treatment, the number of Treg cells was increased, the number of Th17 cells was decreased, the levels of anti-inflammatory factors IL-10 and TGF- $\beta$ were increased, and levels of pro-inflammatory factors IL-17, IL-21 and IL-22 were decreased in chronic hepatitis B patients combined with thrombocytopenia, indicating the decreased autoimmune response and improved thrombocytopenia. The changes were closely related to the complete response.
\end{abstract}

Correspondence to: Dr Yanhua Su, Department of Hematology, The First Affiliated Hospital of Harbin Medical University, 23 Youzheng Street, Nangang, Harbin, Heilongjiang 150036, P.R. China

E-mail: suyanhua1287431@163.com

Key words: chronic hepatitis $\mathrm{B}$, thrombocytopenia, regulatory T cells, Th17 cells

\section{Introduction}

As a common complication in patients with chronic hepatitis $\mathrm{B}$, thrombocytopenia (platelet count $<100 \times 10^{9} / 1$ ) occurs in $76 \%$ of these patients (1). Effects of mild to moderate thrombocytopenia on chronic hepatitis B treatment is generally light without causing spontaneous bleeding, but severe thrombocytopenia can significantly increase the risk of spontaneous bleeding in clinical practice, such as cerebral hemorrhage or gastrointestinal bleeding (2). The incidence of thrombocytopenia is affected by a variety of factors, including inhibition of the production of platelets by bone marrow and decreased activity of thrombopoietin (TPO) (3). In addition, increased intracarotid platelet damage, autoantibodies produced in spleen and blood dilution can also cause thrombocytopenia $(4,5)$.

Autoimmunity is a major immunological factor leading to thrombocytopenia, and studies have reported that T-cell immunity plays an important role in autoimmunity (6). As CD4 ${ }^{+}$ and $\mathrm{CD} 25^{+} \mathrm{T}$ cells, regulatory $\mathrm{T}$ (Treg) cells can inhibit $\mathrm{T}$ cell proliferation and effector function $(7,8)$. Treg can inhibit antigen presenting cells to present antigens to $\mathrm{T}$ cells by secreting IL-10, which in turn inhibit T cell immune response (9). Treg can also secrete TGF- $\beta$ to inhibit the function of T cells and the production of interferon $\gamma($ IFN- $\gamma$ ), thus maintaining a chronic persistent infection of hepatitis B virus (10-12). IL- 6 and TGF- $\beta$ can induce the production of Th17 cells, which can promote the development of inflammatory responses by secreting IL-17, IL-21 and IL-22, so as to promote the development of inflammatory responses (13). Percentage of Th17 cells and serum IL-17 levels were significantly elevated in patients with autoimmune diseases such as rheumatoid arthritis, asthma, and systemic lupus erythematosus (14).

This study showed that Treg cells were closely related to the differentiation process of Th17 cells, and these factors antagonize each other in immune response. Thus, the balance of Treg/Th17 is the key in maintaining immune homeostasis, and the imbalance of Treg/Th17 is associated with a variety of autoimmune diseases (15-17). TGF- $\beta$ is the most important cytokine that affects the differentiation of Treg cells and Th17 cells. Low levels of TGF- $\beta$ and IL-10 can induce the expression of transcription factor $\mathrm{ROR} \gamma$, so as to induce the differentiation 
of T cells to Th17, and high levels of expression of TGF- $\beta$ and IL-10 can induce expression of transcription factor Foxp3, which in turn induce the differentiation of T cells to Treg (18). Previous studies have reported abnormalities in $\mathrm{T}$ cell function in patients with thrombocytopenia $(3,4)$, suggesting that Treg/Th17 cell imbalance may be involved in the pathogenesis of thrombocytopenia. To this end, we investigated the relative levels of Treg cells and Th17 cells in the blood of patients with chronic hepatitis B with thrombocytopenia before and after treatment. In addition, serum levels of Treg cell function related factors IL-10, TGF- $\beta$, and Th17 cell function related factors IL-17, IL-21 and IL-22 were also measured to test whether treatment can restore the balance of Treg/Th17 and provide a reference for evaluating the therapeutic effect.

\section{Patients and methods}

General information. In this study, 45 patients with chronic hepatitis B combined with thrombocytopenia (26 males and 19 females, mean age $44.1 \pm 13.5$ years, mean duration of chronic hepatitis B $12.7 \pm 8.3$ years, all $\mathrm{HBsAg}^{+}$, total bilirubin $>17.1 \mu \mathrm{mol} / 1$, HBV DNA $4.8-12.1 \times 10^{7}$ copies $\left./ \mathrm{ml}\right)$ were selected in Heilongjiang Provincial Hospital (Harbin, China) from June 2015 to December 2016. This study was approved by the Ethics Committee of Heilongjiang Provincial Hospital. Signed informed consents were obtained from all participants before the study. All patients were treated with prednisone acetate tablets (SFDA approval no. H12020689; Tianjin Tianyao Pharmaceuticals Co., Ltd., Tianjin, China) and intravenous injection of immunoglobulin (SFDA approval no. S19994004; Shanxi Kangbao Biological Products Co., Ltd., Changzhi, China) for 60 days.

Sample collection. Fasting venous blood $(5 \mathrm{ml})$ was extracted from each patient before and after treatment. The non-anticoagulated blood was kept at $4^{\circ} \mathrm{C}$ until coagulation. Centrifugation at $8,000 \mathrm{x} \mathrm{g}$ for $15 \mathrm{~min}$ was performed to collect serum to detect cytokines. In addition, heparin anticoagulant blood $(5 \mathrm{ml})$ was also collected and gradient centrifugation was performed to isolate peripheral blood mononuclear cells (PBMC). Cell density was adjusted to $2 \times 10^{6} / \mathrm{ml}$ for the detection of Treg and Th17 cells.

Routine blood examination and liver function tests. Fasting peripheral venous blood was extracted from each patient on the day of admission for routine blood examination and liver function tests including alanine aminotransferase (ALT), aspartate aminotransferase (AST) and prothrombin activity (PTA). After treatment, platelet count returned to normal levels (100-300x10\%/l) was defined as complete response. Platelet count lower than normal level was defined as non-response.

Detection of Treg cell ratio in peripheral blood. Anticoagulant blood $(0.1 \mathrm{ml})$ was transferred to centrifuge tube, and Ficoll lymphocyte separation solution was used to separate lymphocytes. Mouse anti-human CD4-FITC and CD25-PE antibodies (1:1,000; cat. nos. sc-1176 and sc-19628; Santa Cruz Biotechnology, Inc., Dallas, TX, USA) were added, followed by incubation at room temperature for $30 \mathrm{~min}$. After washing with $0.05 \%$ PBST 3 times, $0.1 \mathrm{ml}$ of $0.1 \%$ Triton $\mathrm{X}-100$ was

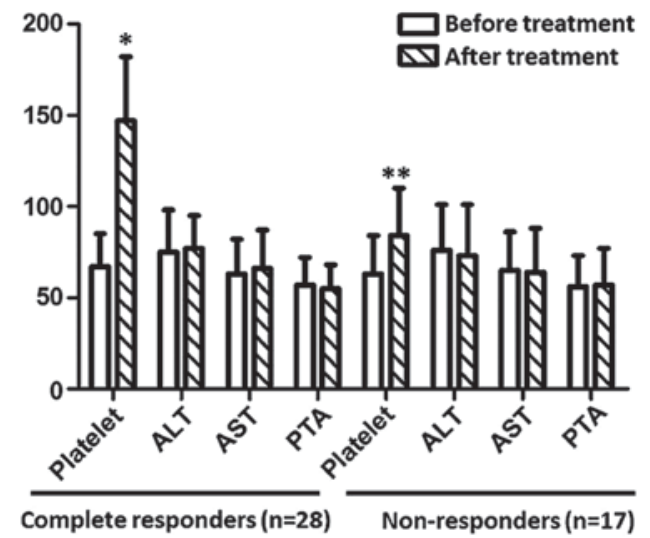

Figure 1. The results of routine blood examination and liver function tests before and after treatment (mean \pm standard deviation). Platelet, x10 $/ 1$; ALT, $\mu / 1$; AST, $\mu / 1$; PTA, $\%$. ${ }^{*} \mathrm{P}<0.01 ;{ }^{* *} \mathrm{P}<0.05$. ALT, alanine aminotransferase; AST, aspartate aminotransferase; PTA, prothrombin activity.

added and incubated for $15 \mathrm{~min}$. Then mouse anti-human Foxp3-PEcy5 monoclonal antibody (1:800; cat. no. sc-53876; Santa Cruz Biotechnology) was added and incubated at room temperature for $30 \mathrm{~min}$. PEcy5-IgG1 was used as control. The percentage of $\mathrm{CD}^{+}, \mathrm{CD}_{2} 5^{+}$and Foxp3 $3^{+}$cells in $\mathrm{CD} 4^{+}$ cells was detected by flow cytometry (BD FACSCanto II; BD Biosciences, San Jose, CA, USA). FlowJo 7.0 software was used to analyze flow cytometry data.

Detection of Th17 cell ratio in peripheral blood. Anticoagulant blood $(0.1 \mathrm{ml})$ was transferred to centrifuge tube, and then Ficoll lymphocyte separation solution was added to separate lymphocytes. Mouse anti-human CD4-FITC antibody was added, followed by incubation at room temperature for $30 \mathrm{~min}$. After washing with $0.05 \%$ PBST 3 times, $0.1 \mathrm{ml}$ of $0.1 \%$ Triton $\mathrm{X}-100$ was added and incubated for $15 \mathrm{~min}$. Then mouse antihuman IL17-PE antibody (1:900; cat. no. sc-376374; Santa Cruz Biotechnology) was added and incubated at room temperature for $30 \mathrm{~min}$. PE-IgG1 was used as control. The percentage of $\mathrm{CD}^{+}$and $\mathrm{IL}-17^{+}$cells in $\mathrm{CD} 4^{+}$cells was detected by flow cytometry (BD FACSCanto II; BD Biosciences). FlowJo 7.0 software was used to analyze flow cytometry data.

Detection of cytokines. Levels of IL-10 (pg/ml), TGF- $\beta(\mathrm{pg} / \mathrm{ml})$, IL-17 (pg/ml), IL-21 (pg/ml) and IL-22 (pg/ml) in serum of patients were measured before and after treatment using a kit (Invitrogen; Thermo Fisher Scientific, Inc., Waltham, MA, USA). Double antibody sandwich ELISA was used. ELISA plate was coated with corresponding mouse anti-human IL-10, TGF- $\beta$, IL-17, IL-21 and IL-22 IgG monoclonal antibodies and mouse IgGl (1:500; cat. nos. sc-365858, sc-65378, sc-53937, sc-137120, sc-134366 and sc-2025; Santa Cruz Biotechnology) was used as a control. A total of $0.1 \mathrm{ml}$ serum was added and incubated at room temperature for $30 \mathrm{~min}$. After washing with $0.05 \%$ PBST 3 times, horse anti-mouse secondary polyclonal antibody IgG-HRP (1:1,000; cat. no. 7076; Cell Signaling Technology, Inc., Danvers, MA, USA) was added and incubated at room temperature for $30 \mathrm{~min}$. After washing with $0.05 \%$ PBST 3 times, enzyme reaction substrate TMB $(0.1 \mathrm{ml})$ was added and incubated for $10 \mathrm{~min}$. Duplicate wells were set for each sample. OD value at $490 \mathrm{~nm}$ was measured by a 


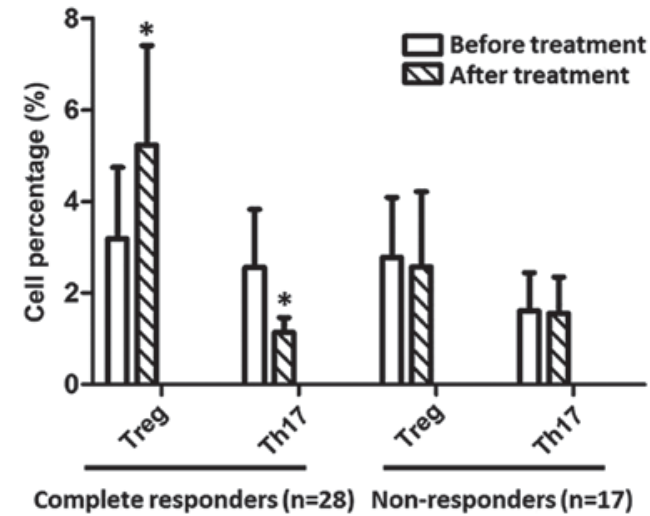

Figure 2. The percentages of Treg cells and Th17 cells in peripheral blood before and after treatment $\left(\%\right.$, mean \pm standard deviation). ${ }^{*} \mathrm{P}<0.01$. Treg, regulatory $\mathrm{T}$.

microplate reader (Multiskan FC; Thermo Fisher Scientific, Inc.) and results were expressed as the mean values of two duplicate wells.

Statistical analysis. Statistical analysis was performed using SPSS 19.0 software (IBM Corp., Armonk, NY, USA). The measurement data were expressed as mean \pm standard deviation, and comparisons between different time-points in the same group were performed using paired t-test, and comparison between groups were performed using Student's t-test. ANOVA was used for comparison between multiple groups and the post hoc test was SNK test. $\mathrm{P}<0.05$ was considered to indicate a statistically significant difference.

\section{Results}

Results of routine blood examination and liver function tests. As shown in Fig. 1, after treatment, platelet count returned to normal level in complete responders $(n=28)$. Although platelet count was significantly increased in nonresponders $(\mathrm{n}=17)(\mathrm{P}<0.05)$, the level is still lower than normal level $\left(100 \times 10^{9} / 1\right)$. After treatment, no significant changes in ALT, AST and PTA were observed in complete responders or non-responders $(\mathrm{P}>0.05)$.

Percentages of Treg and Th17 cells in $\mathrm{CD}^{+} \mathrm{T}$ cells. As shown in Fig. 2, before treatment, the percentage of Treg cells was higher in complete responders than that in non-responders, but the difference was not significant, but the percentage of Treg cells was significantly higher in complete responders than that in non-responders $(\mathrm{P}<0.05)$. After treatment, the percentage of Treg cells was significantly increased and the percentage of Th17 cells was significantly decreased in complete responders $(\mathrm{P}<0.01)$. No significant changes in the percentage of Treg cells and Th17 cells were found in non-responders after treatment $(\mathrm{P}>0.05)$. Compared with non-responders, the percentage of Treg cells was significantly increased and percentage of Th17 cells was significantly decreased in complete responders after treatment $(\mathrm{P}<0.01)$. Repsresentative flow cytomerty is shown in Figs. 3 and 4.
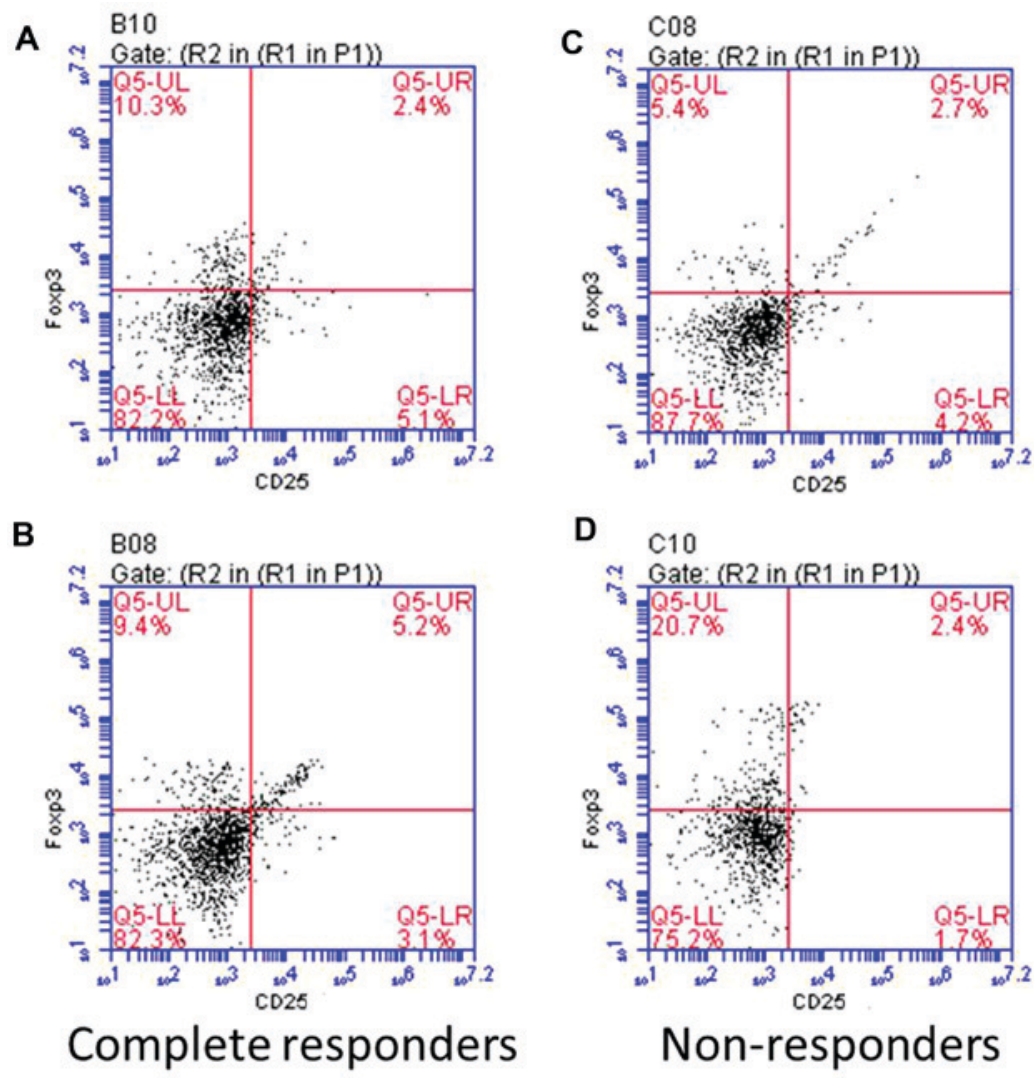

D $\mathrm{C} 10$

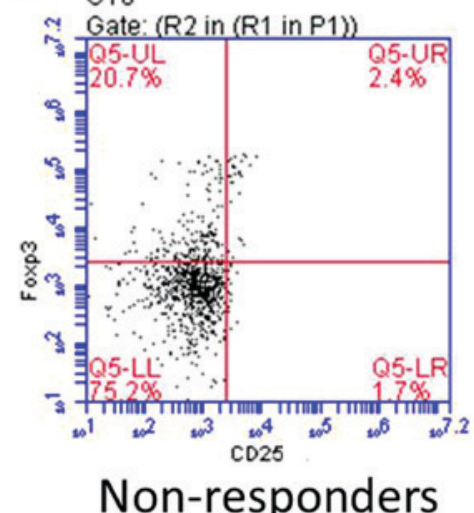

Figure 3. The percentages of Treg cells in peripheral blood before and after treatment. (A and C) Before treatment; (B and D) after treatment. In CD4+ T cells, $\mathrm{CD}_{25} 5^{+}$and Foxp $3^{+} \mathrm{T}$ cells were Treg cells. Treg, regulatory $\mathrm{T}$. 

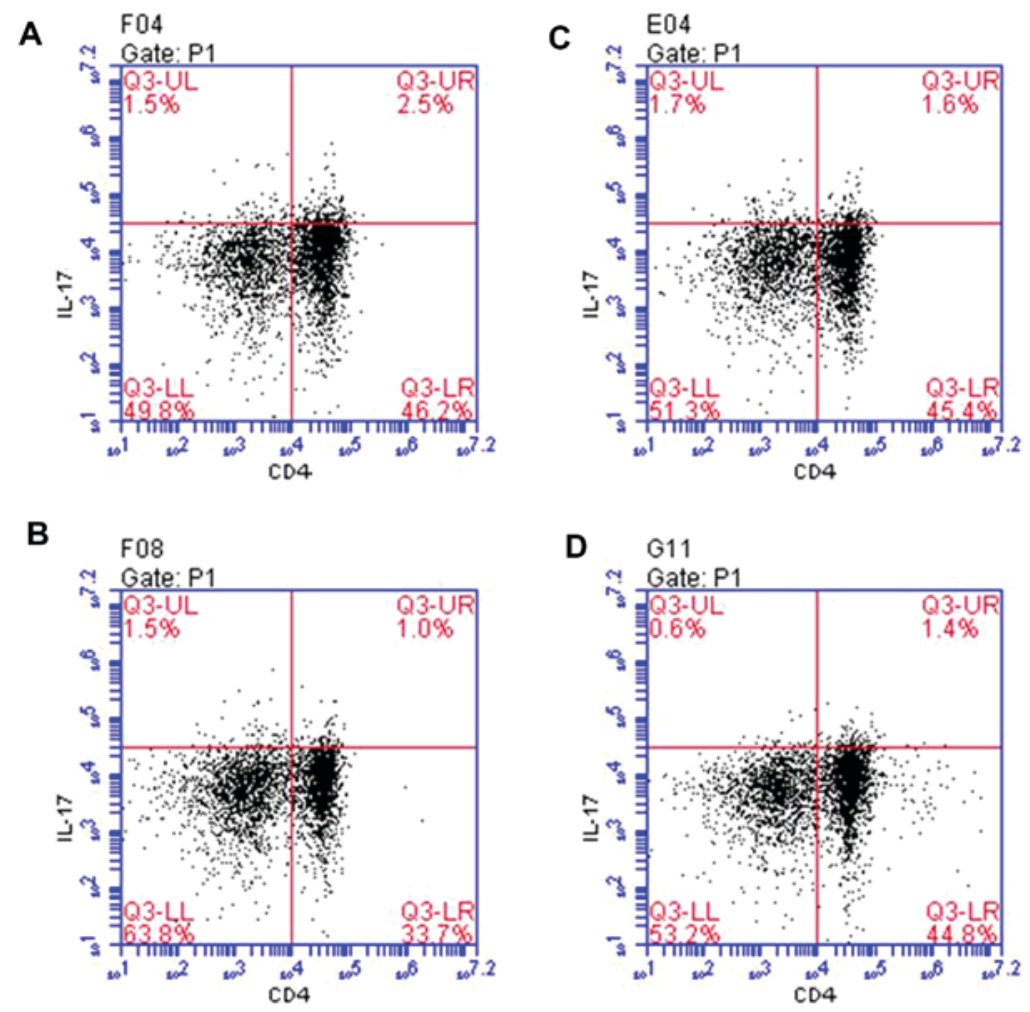

\section{Complete responders}

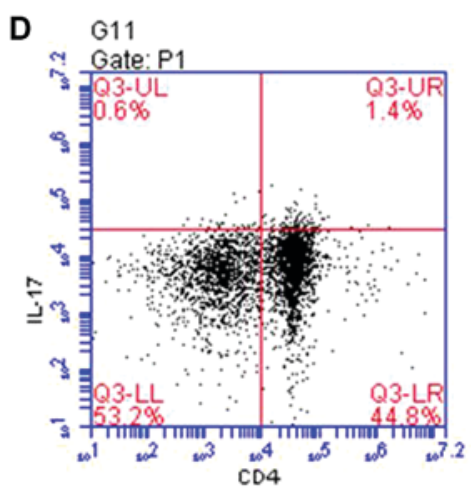

\section{Non-responders}

Figure 4. The percentages of Th17 cells in peripheral blood before and after treatment. (A and C) Before treatment; (B and D) after treatment. CD4 ${ }^{+}$and IL-17 $\mathrm{T}$ cells were Th17 cells.

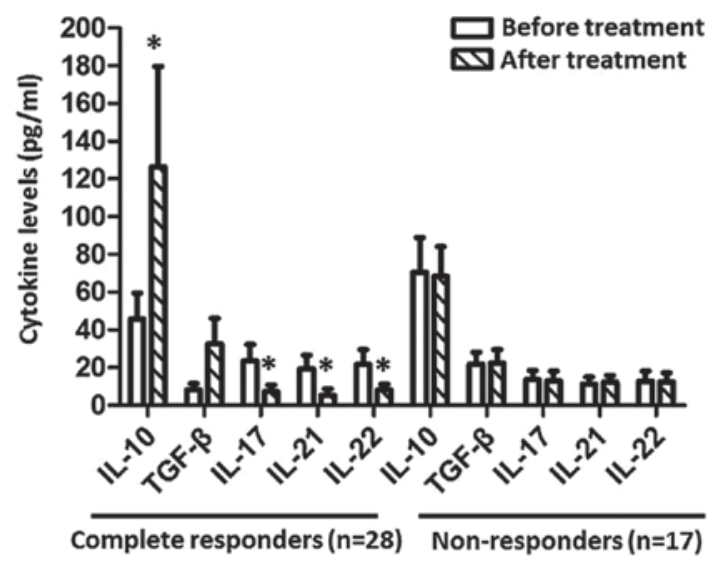

Figure 5. The serum levels of cytokines before and after treatment $(\mathrm{pg} / \mathrm{ml}$, mean \pm standard deviation). ${ }^{*}<<0.01$.

Serum levels of cytokines in patients before and after treatment. As shown in Fig. 5, before treatment, levels of IL-10 and TGF- $\beta$ were significantly lower, and levels of IL-17, IL-21 and IL-22 were significantly higher in complete responders than those in non-responders $(\mathrm{P}<0.05)$. After treatment, levels of IL-10 and TGF- $\beta$ were significantly increased, and levels of IL-17, IL-21 and IL-22 were significantly decreased in complete responders $(\mathrm{P}<0.05)$. No significant differences in levels of IL-10, TGF- $\beta$, IL-17, IL-21 and IL-22 were found in non-responders after treatment. Thus, after treatment, levels of IL-10 and TGF- $\beta$ were significantly higher, and levels of
IL-17, IL-21 and IL-22 were significantly lower in complete responders than those in non-responders $(\mathrm{P}<0.05)$.

\section{Discussion}

Treg cells can maintain the tolerance of autoimmune and anti-inflammatory response. On the contrary, Th17 can mediate autoimmune diseases and inflammatory response. The balance between these two factors can maintain the steady state of immune response, and the imbalance will lead to the occurrence of autoimmune disease. Treg/Th17 cell imbalance is an important mechanism leading to persistent viral infection in patients with chronic hepatitis B. Increase in number of Tregs and decrease in the number of Th17 can lead to inhibition of $\mathrm{T}$ cell immunity in patients, and cellular immune response against hepatitis B virus will be inhibited $(19,20)$. Studies have shown that platelet-related antibodies are one of the major causes of thrombocytopenia (21), suggesting that Treg/Th17 cell imbalance may be involved in the production of autoimmune platelet-associated antibodies (22).

This study showed that the percentage of Treg cells was increased, and percentage of Th17 cells was decreased, and platelet count returned to normal levels in patients with complete response. Correspondingly, levels of IL-10 and TGF- $\beta$ were significantly increased, and levels of IL-17, IL-21 and IL-22 were significantly decreased after treatment, which reflects that Treg cells can inhibit inflammatory response and reduce the number of Th17, which in turn improves thrombocytopenia. No significant changes in Treg, Th17 and related cytokines 
were observed after treatment, indicating that treatment failed to reverse the imbalance of Treg/Th17. Therefore, Treg, Th17 and related cytokines can be used to predict prognosis. It is noteworthy that before treatment, numbers of both Treg cells and Th17 cells were higher in complete responders than those in non-responders, suggesting that the higher percentage of two cells may indicate good treatment outcomes.

Consistent with previous studies $(19,23,24)$, this study showed that recovery of Treg/Th17 cell balance is beneficial for the improvement of thrombocytopenia in patients with chronic hepatitis B. Our study provides a theoretical basis for clinical treatment of thrombocytopenia in patients with hepatitis $\mathrm{B}$.

\section{Acknowledgements}

Not applicable.

\section{Funding}

No funding was received.

\section{Availability of data and materials}

The datasets used and/or analyzed during the current study are available from the corresponding author on reasonable request.

\section{Authors' contributions}

YW and LW collected and analyzed the general information of patients. WG collected the blood and tissue samples. XC performed ELISA. YS helped with flow cytometry. All authors read and approved the final manuscript.

\section{Ethics approval and consent to participate}

This study was approved by the Ethics Committee of Heilongjiang Provincial Hospital (Harbin, China). Signed informed consents were obtained from all participants before the study.

\section{Patient consent for publication}

Not applicable.

\section{Competing interests}

The authors declare that they have no competing interests.

\section{References}

1. Peck-Radosavljevic M: Thrombocytopenia in liver disease. Can J Gastroenterol 14: 60D-66D, 2000

2. Thomopoulos KC, Labropoulou-Karatza C, Mimidis KP, Katsakoulis EC, Iconomou G and Nikolopoulou VN: Non-invasive predictors of the presence of large oesophageal varices in patients with cirrhosis. Dig Liver Dis 35: 473-478, 2003.

3. Aref S, Mabed M, Selim T, Goda T and Khafagy N: Thrombopoietin (TPO) levels in hepatic patients with thrombocytopenia. Hematology 9: 351-356, 2004.

4. Afdhal N, McHutchison J, Brown R, Jacobson I, Manns M, Poordad F, Weksler B and Esteban R: Thrombocytopenia associated with chronic liver disease. J Hepatol 48: 1000-1007, 2008.
5. Stornaiuolo G, Amato A and Gaeta GB: Adefovir dipivoxil-associated thrombocytopenia in a patient with chronic hepatitis B. Dig Liver Dis 38: 211-212, 2006.

6. Rodeghiero F, Stasi R, Gernsheimer T, Michel M, Provan D, Arnold DM, Bussel JB, Cines DB, Chong BH, Cooper N, et al: Standardization of terminology, definitions and outcome criteria in immune thrombocytopenic purpura of adults and children: Report from an international working group. Blood 113: 2386-2393, 2009.

7. Shevach EM: Mechanisms of foxp $3^{+} \mathrm{T}$ regulatory cell-mediated suppression. Immunity 30: 636-645, 2009.

8. Wing K, Onishi Y, Prieto-Martin P, Yamaguchi T, Miyara M, Fehervari Z, Nomura T and Sakaguchi S: CTLA-4 control over Foxp $3^{+}$regulatory T cell function. Science 322: 271-275, 2008.

9. Mittal SK and Roche PA: Suppression of antigen presentation by IL-10. Curr Opin Immunol 34: 22-27, 2015.

10. Keswani T, Sarkar S, Sengupta A and Bhattacharyya A: Role of TGF- $\beta$ and IL- 6 in dendritic cells, Treg and Th17 mediated immune response during experimental cerebral malaria. Cytokine 88: 154-166, 2016.

11. Liu J, Hong X, Lin D, Luo X, Zhu M and Mo H: Artesunate influences Th17/Treg lymphocyte balance by modulating Treg apoptosis and Th17 proliferation in a murine model of rheumatoid arthritis. Exp Ther Med 13: 2267-2273, 2017.

12. Shrivastava S, TrehanPati N, Patra S, Kottilil S, Pande C, Trivedi SS and Sarin SK: Increased regulatory $\mathrm{T}$ cells and impaired functions of circulating CD8 T lymphocytes is associated with viral persistence in Hepatitis B virus-positive newborns. J Viral Hepat 20: 582-591, 2013.

13. Feng S, Chen XM, Wang JF and Xu XQ: Th17 cells associated cytokines and cancer. Eur Rev Med Pharmacol Sci 20: 4032-4040, 2016.

14. Harrington LE, Hatton RD, Mangan PR, Turner H, Murphy TL, Murphy KM and Weaver CT: Interleukin 17-producing CD4 ${ }^{+}$ effector $\mathrm{T}$ cells develop via a lineage distinct from the T helper type 1 and 2 lineages. Nat Immunol 6: 1123-1132, 2005.

15. Li J, Lai X, Liao W, He Y, Liu Y and Gong J: The dynamic changes of Th17/Treg cytokines in rat liver transplant rejection and tolerance. Int Immunopharmacol 11: 962-967, 2011.

16. Ni K, Zhao L, Wu J, Chen W, HongyaYang and Li X: Th17/Treg balance in children with obstructive sleep apnea syndrome and the relationship with allergic rhinitis. Int J Pediatr Otorhinolaryngol 79: 1448-1454, 2015.

17. Zheng Y, Wang Z, Deng L, Zhang G, Yuan X, Huang L, Xu W and Shen L: Modulation of STAT3 and STAT5 activity rectifies the imbalance of Th17 and Treg cells in patients with acute coronary syndrome. Clin Immunol 157: 65-77, 2015.

18. Kleinewietfeld M and Hafler DA: The plasticity of human Treg and Th17 cells and its role in autoimmunity. Semin Immunol 25: 305-312, 2013.

19. Su ZJ, Yu XP, Guo RY, Ming DS, Huang LY, Su ML, Deng Y and Lin ZZ: Changes in the balance between Treg and Th17 cells in patients with chronic hepatitis B. Diagn Microbiol Infect Dis 76: 437-444, 2013.

20. Li K, Liu H and Guo T: Th17/Treg imbalance is an indicator of liver cirrhosis process and a risk factor for HCC occurrence in HBV patients. Clin Res Hepatol Gastroenterol 41: 399-407, 2017.

21. Gilli SC, de Souza Medina S, de Castro V, Fernandes LG and Saad ST: Platelet associated IgG may be related with thrombocytopenia in patients with myelodysplastic syndromes. Leuk Res 36: 554-559, 2012.

22. Aboul-Fotoh L-M, Abdel Raheem MM, El-Deen MA and Osman AM: Role of $\mathrm{CD} 4^{+} \mathrm{CD} 25^{+} \mathrm{T}$ cells in children with idiopathic thrombocytopenic purpura. J Pediatr Hematol Oncol 33: 81-85, 2011.

23. Liu B, Gao W, Zhang L, Wang J, Chen M, Peng M, Ren H and $\mathrm{Hu} \mathrm{P}$ : Th17/Treg imbalance and increased interleukin-21 are associated with liver injury in patients with chronic severe hepatitis B. Int Immunopharmacol 46: 48-55, 2017.

24. Cao J, Chen C, Zeng L, Li L, Li X, Li Z and Xu K: Elevated plasma IL-22 levels correlated with Th1 and Th22 cells in patients with immune thrombocytopenia. Clin Immunol 141: 121-123, 2011. 